DOI: 10.21767/2472-1948.100011

\title{
Transdermal Drug Delivery (TDD) through Skin Patches
}

Received: May 16, 2016; Accepted: May 16, 2016; Published: May 19, 2016

Metrology defines the standards of measurements that are utilized in all fields of science, technology, engineering, mathematics and manufacturing (STEMM) and provides control for comparisons. Prescribing such a standard for manmade apparatuses is mostly straight forward or, in indirect situations, may require regressive analysis. In drug administration systems, the development of such standards is highly desirable because human life is on the line and even miniscule errors have detrimental outcomes. Ironically, the high urgency in the development of physiological standards is at best empirically derived from invasive tests and represented by model approximations. This error is due to the individuality of different members of the population, which stems from genetics, body weight, lifestyle, age and climate. Therefore, most metrological standards for pharmaceuticals are set up as a range that requires additional reinforcement from clinical trials. Despite the ultimate reliance on clinical trials and associated model error, computational analysis in the form of statistical designs is useful and provides for a safer platform for clinical trials. This study will focus on Transdermal Drug Delivery (TDD), which is quite underused in the market and is lacking in computational investigation, despite its clear advantages.

TDD through skin patches is both economically and pharmacokinetically promising. The TDD market was valued at approximately $\$ 27$ billion in 2013 and was predicted to experience an $8 \%$ Compound Annual Growth Rate (CAGR) for the five years following the publication of this report [1]. Over the years, local anesthetic patches have undergone several upgrades, despite their constituting only $2 \%$ of the global TDD market [2]. Part of the interest in investment into local pain relief patches is to control the usage of such therapeutics, as they contain controlled substances that may cause addiction. Furthermore, slow release patches are more effective than bolus doses that fluctuate.

Targeted drug delivery and sustained release drugs (Figure 1) are shown in the bar graph for market value of oral drugs. Sustained release drugs do not solely represent skin patches, which explain the increased value of the market sector, compared to the value reported in the previous paragraph. The key growth trends observed in this depiction between the years, 2004-2011, are as follows: a $2.5 x$ increase for transmucosal drug delivery, a $5 x$ increase for targeted drug delivery and a $1.8 x$ increase for sustained release, implants and TDD. The former value can seem unnerving, compared to the increase in value associated with targeted drug delivery. This drastic difference is due to several
Natali R Gendellberg, Roberto Falcone and Nuggehalli M Ravindra

\author{
Interdisciplinary Program in Materials \\ Science \& Engineering, New Jersey Institute \\ of Technology, Newark, NJ, USA
}

Corresponding author: Nuggehalli M Ravindra

n.m.ravindra@njit.edu

Interdisciplinary Program in Materials Science \& Engineering, New Jersey Institute of Technology, USA.

Tel: 9735963278

Citation: Gendelberg NR, Falcone F, Ravindra NM. Transdermal Drug Delivery (TDD) Through Skin Patches. J Sci Ind Metrol. 2016, 1:2.

factors. First, targeted drug delivery technologies experienced a significant breakthrough between the years 2006-2011, before which sustained release, implants and TDD dominated the therapeutics market as its primary constituent. Second, sustained release, implants and TDD still encompass almost half of the therapeutics market. Third, many targeted drug delivery technologies utilize TDD and sustained drug release. Therefore, the extreme increase could be due to intermarket overlap. The increase in market value of transmucosal therapies does not signify a potential profit. The drug market percentage represented by transmucosal drugs decreased by $2 \%$ and began at a substantially lower value compared to other therapies.

Transdermal drug delivery has a slow and continuous drug release profile. This sort of profile is ideal for treatments involving drugs with a short half-life that must be administered frequently over long periods of time. The constant presence of the patch also ensures timely dosages that prevent unwanted variations in the drug concentration in the blood.

The large surface area of the skin makes skin patches a highly accessible, yet non-invasive and kinetically maneuverable form of treatment. As can be seen in Figure 2, oral and bolus drug administration involve fluctuations of the drug concentration in the blood, with a spike at the time of administration and a 


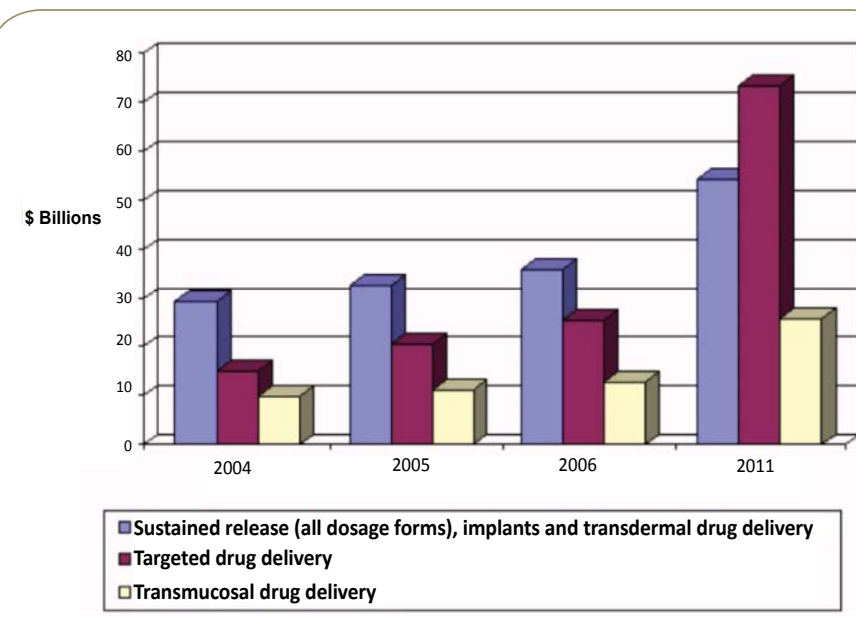

Figure 1 Drug delivery market trends [2].

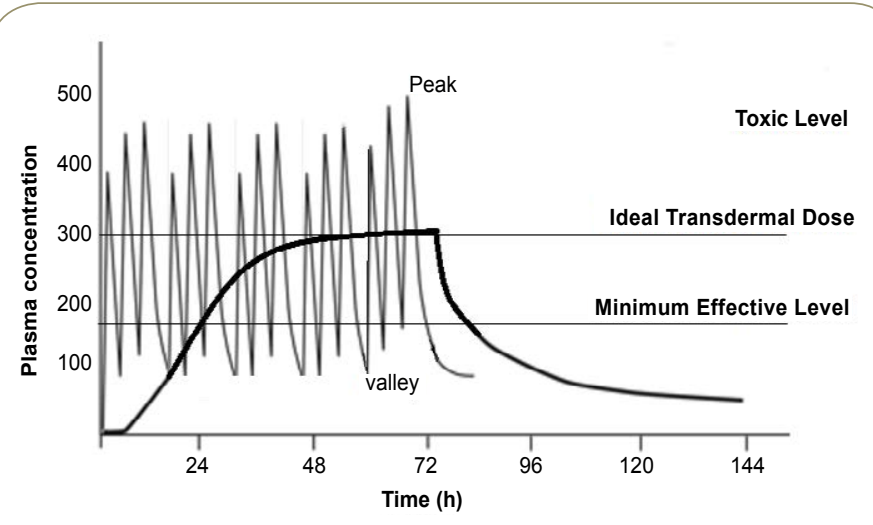

Figure 2 Cycle of blood concentration with respect to time in TDD.

sudden decrease at peak concentration. In order to obtain an average dosage around the middle of the therapeutic range, the initial dosage must be much higher. Each valley-to-valley segment in Figure $\mathbf{2}$ represents a single administration. From this representation, it is evident that such treatment involves cyclical dosage fluctuations whose range varies between individuals based on weight, age, life-style and genetics. Therefore, for different individuals, the valleys and peaks of the drug administration may exceed or fall below the toxic and minimal therapeutic drug concentrations, respectively [3].

The oral/bolus forms of drug administration set up a precedent where a patient is at risk or is not receiving effective treatment at a certain fixed time, which can also bring upon a set of side effects such as nausea. In contrast, the skin patch maintains a quite consistent dosage that can be tailored by modification of the polymer matrix through which the drug diffuses. Application time of the patch is determined based on the point at which the drug remaining in the patch reservoir provides for the determined margin of decrease in resulting blood concentration. Furthermore, administration can be instantaneously halted via patch removal in the case of evident deleterious side effects.

The ability of the skin patch to circumvent passage through the gastrointestinal tract (GIT) has two advantages: 1) the drug does not damage the GIT or its mucus membrane and 2) the "First Pass Effect" is eliminated. The "First Pass Effect" is a result of the body's clearance system. After entering the stomach, the drug may be detected and cleared out by the body as a foreign object in passage through the intestines and the liver. This results in a reduced dose entering the blood stream where the drug undergoes another series of clearance. This requires that the initial dosage administered to the patient be much higher than the therapeutic dosage. As the amount of drug cleared out varies between individuals, this may result in varying side effects that cannot be halted without complete clearance that is physiologically controlled [4].

The challenge for metrological design of skin patches stems from the skin's foundation, which is built on preventing the entrance of any foreign objects into the body. As such, the charge and " 500 $\mathrm{Da}$ " size limitations, eliminate the design of skin patches for a large scope of therapeutics, including: gene therapy, liposomal drug delivery and charged particles. As an individual's lifestyle impacts metabolic activity, while stratum corneum (SC) thickness and skin composition varies in different parts of the body and between individuals, the theoretical blood dosage with respect to polymer matrix design varies between individuals and is highly reliant on clinical trials. As a result, skin patch dosages are determined based on clinical trial results and thus pose high risk to the subject because the resulting dosage may fall in an individual's toxic dosage. Finally, skin adhesives may cause skin irritation and rash $[5,6]$. As the SC thickness varies, the diffusion path length used in the governing diffusion equations is variable as well.

The two primary types of skin patches are: the reservoir patch and the matrix patch. The main difference between these types of patches is their form of drug storage and kinetics. The patch acts as an initial drug reservoir. Once the patch is placed at the site of application, the drug begins to diffuse into the SC due to osmotic pressure and forms a secondary reservoir in the SC, which further emphasizes the SC's rate limiting property. The drug diffuses through the SC into capillaries, present in the hypodermis and thereby enters the blood stream.

As the polymer membrane through which the drug active diffuses in the patch is not a fluid, the Stokes-Einstein parameters are breached. Furthermore, the cell membrane exhibits anisotropic diffusion/penetration properties. The fluid in the plane of the membrane results in transverse particle diffusion through the fluid. In one form of diffusion through the SC, lateral diffusion or transmembrane diffusion, the diffusing particle is set back by the hydrocarbon chains that are vertically anchored. In the second form of diffusion through the SC matrix (around the corneocytes), there are physical hindrances that lead to loss of energy to the particle that results in a smaller diffusivity. The SC is the rate limiting membrane in the outermost position of the epidermis. It is made up of lipids with embedded corneocytes, which are dead cells filled with keratin. The "brick and mortar" structure of the SC (Figure 3) prevents transmembrane diffusion and facilitates increased diffusion path length. Therefore, in both processes of diffusion through the skin patch membrane and the skin, the diffusion of the drug is Non-Stokesian [7]. 
The key to metrological design of TDD systems via skin patches is the computation of drug diffusivity through the polymer matrix in the skin patch. Once the diffusivity is computed, an initial dosage can be determined to achieve the standard therapeutic blood concentration. The diffusivity through the polymer membrane relies on the morphology of the drug, the available volume for diffusion based on the polymer characteristics and the thickness of the membrane. These design parameters are held as standard for therapeutics and may be optimized based on the metrological diffusivity computations.

Non-Stokesian diffusivity computations vary based on diffusion characteristics. They normally involve adding an exponential multiplier to the Stokes-Einstein equation that accounts for the system's deviation from Stokesian Diffusion (Equation 1). In the case of particles diffusing through a polymer membrane and the SC, analysis via the statistical free volume theory is most adequate. This analysis involves an exponential factor of the product of the overlap factor of the shared free volume $(\varepsilon)$ and the ratio of the critical free volume and total free volume. This portion represents the probability function that computes the probability of the diffusant encountering a hole with the minimum critical volume required for diffusion to occur. In this view, the particle and the hole are viewed as mutually diffusing. Experiments performed to assess free hole volume in a hydrocarbon chain matrix, which are comparable to cell membrane hydrocarbon tails, showed that approximately $35 \%$ of the total volume is attributed to free hole volume [8].

$D_{\infty}=D_{0} e^{-E / k T} e^{-\varepsilon V^{*} / V}$

$\varepsilon=\frac{\hat{V}_{1}}{\hat{V}_{2}}$

The basic probability function for diffusion through a polymer matrix is that posed by Vrentas and Dudas for the infinite particle diffusivity, as can be seen in equation 1 . The term $D_{0}$ refers to the Stokes-Einstein diffusivity. The first exponential term accounts for the Arrhenius factor of the diffusion that is related to the kinetic energy of the diffusing particle. $E$ refers to the activation energy required for the drug to overcome the attractive intermolecular forces with its medium in order to diffuse. T refers to the temperature in Kelvin and $\mathrm{k}$ is the Boltzmann constant. The second exponential term accounts for the ratio of the critical jumping volume required for diffusion with respect to the total available free volume $\left(\mathrm{V}^{*} / \mathrm{V}\right)$ multiplied by the ratio of the solvent to polymer jumping unit that is determined by a factor of the occupied volume of the diffusing molecule (subscript 1 ) and the polymer matrix (subscript 2).

It is not feasible to computationally calculate the exact free hole volume in a polymer matrix as it accounts for the difference between the total volume of the matrix and the Van der Waals volume of the polymers, which may vary with time as well. Therefore, the free hole volume should be statistically based on the following premises: (1) it is most probable to encounter small holes and (2) the formation of large holes is highly improbable. Thus, the diffusion coefficient depends on the number of holes, the net hole formation frequency and the probability of finding a hole as a function of the exponent of the ratio of the minimum hole volume necessary for diffusion with respect to the average hole volume [7].

Table 1 lists some of the TDD patches designed since 1979. The dosage determinations and efficacy analyses performed for these products are highly reliant on clinical trials and subject responses to questionnaires, which are highly qualitative and cannot be used as a valid standard for comparison. Generally speaking, due to skin variations, a specific standard cannot be proposed for skin patches as such a value varies between individuals. The resulting death of a skin patch contraceptive user, a few years ago, and the appearance of blood clots in other females that used this contraceptive, emphasizes the need for placement of individual design specifications and their detection prior to clinical trials [5]. These side effects were unforeseen prior to FDA approval, as the clinical trials could not predict that the temporary increase in skin

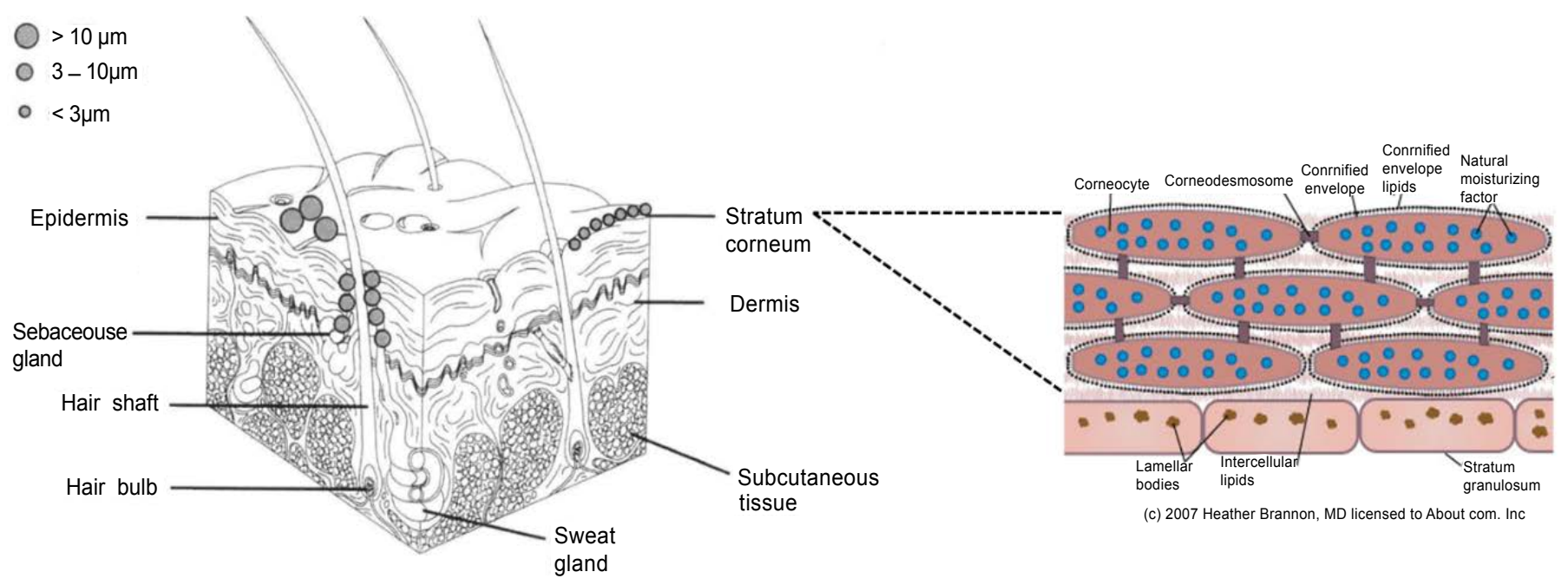

Figure 3 Skin anatomy with a close up of the SC and drug diffusion paths in the inset [15]. 
temperatures for athletic individuals would result in toxic blood levels of the drug.

Falcone et al. [6] modified the Duda-Zalinsky equation (DZE) for diffusivity through a polymer membrane, so as to compute the diffusivity of nicotine as it diffuses through the polymer membrane in the nicotine patch. The resulting diffusivity was of the same order of magnitude as nicotine diffusivity values reported in the literature, with a $17 \%$ error with respect to diffusivity values obtained from diffusion analysis in a Franz Cell apparatus (Figure 4). In this apparatus, the patch is clamped between the cell top and cell body, which is in contact with constantly stirred saline in its primary chamber and has water flowing through the outer wall of the primary chamber so as to control the system temperature. Samples extracted periodically from the primary chamber are used to obtain the drug accumulation with respect to time to solve for the diffusivity. In order to maintain the patch in constant contact with the cell media, it is important to maintain the media volume constant by adding back the same volume of media removed during sampling. Additionally, this ensures accurate evaluation of system accumulation. Diffusion tests run on the Franz Cell

Table 1 Marketed patches, with emphasis on the evolved lidocaine patches [17].

\begin{tabular}{|c|c|c|}
\hline Year & Generic (Brand) Names & Indication \\
\hline 1979 & Scopolamine (Transdenn Scope) & Motion sickness \\
\hline 1984 & Clonidine (Catapress TTS4P) & Hypertension \\
\hline 1986 & Estradiol (Estraderm $\left.{ }^{\circ}\right)$ & Menopausal symptoms \\
\hline 1990 & Fentanyl (Duragesic ${ }^{\circ}$ ) & Chronic pain \\
\hline 1991 & $\begin{array}{c}\text { Nicotine (Nicoderme Habitrole } \\
\text { Prostep }{ }^{\circ} \text { ) }\end{array}$ & Smoking cessation \\
\hline 1993 & Testosterone (Androderm ${ }^{\circ}$ ) & Testosterone deficiency \\
\hline 1995 & $\begin{array}{l}\text { Lidocaine/epinephrine } \\
\text { (lontotainetle) }\end{array}$ & Local dermal analgesia \\
\hline 1998 & $\begin{array}{l}\text { Estradiol/norethindrone } \\
\left.\text { (Combipatch }{ }^{\circ}\right)\end{array}$ & Menopausal symptoms \\
\hline 1999 & Lidocaine (Lidoderm ${ }^{\circ}$ ) & Post-herpetic neuralgia pain \\
\hline 2001 & $\begin{array}{c}\text { Ethinyi estradiol/norelgestromin } \\
\left.\text { (OrthoEvra }{ }^{\circ}\right)\end{array}$ & Contraception \\
\hline 2003 & $\begin{array}{l}\text { Estradiol/levonorgestrel } \\
\text { (Climara Pure) }\end{array}$ & Menopause \\
\hline 2003 & Oxybutynin (Oxytrol $\left.{ }^{\circ}\right)$ & Overactive bladder \\
\hline 2004 & Lidocaine/ultrasound (SonoPrepO) & Local dermal anesthesia \\
\hline 2005 & LIdocaine/tetracalne (Synera) & Local dermal analgesia \\
\hline 2006 & FentanyViontophoresis (lonsyse)" & Acute postoperative pain \\
\hline 2006 & Methylphenidate (Daytrana ${ }^{\circ}$ ) & ADHD \\
\hline 2006 & Selegiline (Emsam ${ }^{\circ}$ ) & Depression \\
\hline 2007 & Rotigotine (Neuproe)" & Parkinson's disease \\
\hline 2007 & Rivastigmine (F.xelon ${ }^{\circ}$ ) & Dementia \\
\hline 2008 & Granisetron (Sancuso ${ }^{\circ}$ ) & Chemo-Induced emesis \\
\hline 2009 & Oxybutynin (Gelnique") & Overactive bladder \\
\hline 2010 & Buprenorphine (Butranse) & Chronic pain \\
\hline
\end{tabular}

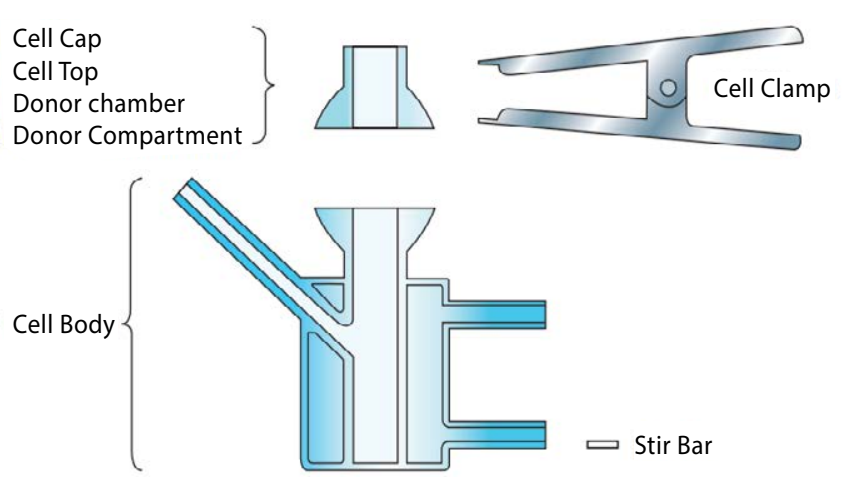

Figure 4 Components of PermGear's Franz Cell apparatus [16].

apparatus are fitting for setting up standards for clinical trials, as the system may be designed to test the diffusivity of the skin patch on its own and in combination with a layer of skin below it. The computational analysis, in conjunction with the results from the flow device, proved successful for the nicotine patch without diffusion enhancements. These diffusivity values may be used in the design of future skin patches and as a tentative analysis prior to clinical trials, so as to set valid standards.

TDD enhancement technologies can enhance drug diffusion and targeting either by increasing the SC permeability or by incorporating the drug in a "delivery vehicle". The purpose of investment into TDD penetration enhancement technologies is to improve drug diffusion and to facilitate the utilization of TDD for a wider range of drugs; including those that violate the charge and size barriers posed by the SC. Iontophoresis and thermal ablation are the more promising permeability enhancers. Hypodermic needles that provide direct penetration below the SC have also been devised. However, due to rapid healing of the SC, utilization of these needles is impractical for long-term treatments. Examples of targeting enhancers include liposomal drug delivery or the incorporation of superparamagnetic iron oxide nanoparticles into the drug formulation. Such targeting enhancements may not be used for TDD without a resolution of the barrier posed by the SC, as their construct exceeds the 500 Da molecular weight limit posed by the SC.

An iontophoretic system contains two electrodes that are in contact with the skin and extend out of electrolyte chambers. One electrolyte chamber contains the drug along with anions, while the other chamber contains a buffer solution of similar polarity. A small electric current passing through the electrodes causes an electric potential across the skin. As this functions as an electro-osmotic system, the solvent flows in the direction of the ion movement and transports the cationic drug molecules through the skin. The dosage results from the magnitude of the charge generated by the applied current. This method generates better diffusion outcomes with cationic drugs because the cell membrane potential is negative, with increase in cationic drug contribution to electro-osmosis being directly proportional to the molecular weight of the drug [7].

Despite osmosis generally being a passive form of diffusion, iontophoresis involves convective diffusion as the therapeutics 
are repelled into the skin. This property results in additional functions such as physiological monitoring by attraction of water soluble molecules present in the interstitial fluid. Drug dosages can be fine-tuned to provide for pulsatile drug delivery profiles that may prevent the body from becoming tolerant to the therapeutic [9]. Iontophoresis requires strict current limitation to prevent erythema. This method is not cost effective and was recalled by the European Medicine Agency due to continuous fentanyl release resulting from corrosion products from the system components when the device was deactivated, as well $[10,11]$

Thermal ablation involves the selective removal of the SC without causing permanent damage to the skin and its infrastructure. Heat is applied to the skin by an external heat source for a short amount of time, which disrupts and removes the SC and creates microchannel structures in the skin. The resulting microchannel structures are caused by three processes that occur as a result of the sharp heat exposure: (1) the loss of the structural viability of the brick-and-mortar structure of the SC; (2) the disruption of the keratin network within the SC and (3) the decomposition and vaporization of keratin due to heat, which leaves behind microscale vacancies in the SC [12].

It has been shown that the SC permeability is significantly increased with increasing temperature during thermal ablation, rather than with heating time. This being the case, there must be a strict control over the balance between effective temperature levels and exposure time of the tissue below the SC to prevent permanent damage to the skin. Lee et al. (2011) [13] devised an apparatus to locally heat the skin for duration of the order of $100 \mu \mathrm{s}$. This apparatus consists of water in a micro chamber that is rapidly heated by discharging electric current through it. By utilizing the conversion of electrical current to thermal and mechanical energy in a localized manner, the generated jet of superheated steam is immediately ejected unto the skin [12]. This apparatus provided specific localized removal of the SC and increased its permeability by three to four orders of magnitude.

Detailed research is crucial for further specification and broadening of patch applications to include therapeutics that have charge or are larger than $500 \mathrm{Da}$. Such therapeutics includes liposomal drug delivery, magnetic nanoparticle assisted drug delivery and gene therapy. Converting these treatments into TDD patches will not only alleviate the experience involved with these treatments, but will help to improve their efficacy due to the sustained release feature of the skin patch. To ensure that the aforementioned diffusion enhancers are safely used and properly controlled, proper standards must be quantified for different conditions and for a variety of skin types, age groups and habits. Diffusion enhancers open many therapeutic opportunities; but, without a proper set of controls, they may cause fatalities due to increased drug delivery. It is crucial to develop a metrological procedure for Falcone et al.'s [6] computational analysis to incorporate enhanced skin patches, as well. Recently, research performed on the Synera Heated Lidocaine-Tetracaine patch has been used to assess the application of parameters in the DZE equation to enhanced skin patches. The cohesive energy and viscosity calculations for the diffusants and the polymer membrane provide important information on the diffusivity characteristics of the drug for varying temperature settings [14]. This pre-clinical trial approach, utilizing a scientific approach via computational analysis, serves as a platform for safer clinical trials and for optimized design of diffusion enhancers in the skin patches.

The authors acknowledge with thanks the support of Dr. Sarah Dolan of Galen U.S Inc. 


\section{References}

1 Bhowmik D, Duraivel S, Sampath Kumar K P (2012) Recent Trends in Challenges and Opportunities in Transdermal Drug Delivery System. The Pharma Innovation: 9-23.

2 Sampath Kumar K P, Bhowmik D, Chiranjib B, Chandira R M (2010) Transdermal Drug Delivery System - A Novel Drug Delivery System and Its Market Scope and Opportunities. International Journal of Pharma and Bio Sciences: 1-21.

3 Bolognia J, Jorizzo JL, Schaffer JV (2012) Dermatology. Elsevier Saunders 1: 2065-2073.

4 Pond SM, Tozer TN (1984) First-Pass Elimination. Clinical Pharmacokinetics: 1-25.

5 Roberts J (2006) Women Sue Maker of Birth Control Patch. CBSNews CBS Interactive.

6 Falcone R, Jaffe M, Ravindra NM (2013) New screening methodology for selection of polymeric matrices for transdermal drug delivery devices. Bioinspired, Biomimetic and Nanobiomaterials 2: 65-75.

7 Stein WD, Lieb WR (1986) Transport and Diffusion across Cell Membranes. Orlando Academic 94-102.

8 http://central.gutenberg.org/articles/Joback_method

9 Naik A, Kalia YN, Guy RH (2000) Transdermal Drug Delivery: Over- coming the Skin's Barrier Function. Pharmaceutical Science \& Technology Today 318-326.

10 Paudel KS, Milewski M, Swadley CL, Brogden NK, Ghosh P, et al. (2010) Challenges and Opportunities in Dermal/transdermal Delivery. Therapeutic Delivery 109-131.

11 Bondi A (1954) Free Volumes and Free Rotation in Simple Liquids and Liquid Saturated Hydrocarbons. The Journal of Physical Chemistry J Phys Chem 929-939.

12 Binghe W, Hu L, Siahaan T (2005) Drug Delivery: Principles and Applications. Wiley.

13 Lee J W, Priya G, Jung-Hwan P, Allen M G, Prausnitz M R (2011) Microsecond Thermal Ablation of Skin for Transdermal Drug Delivery. Journal of Controlled Release 154.1: 58-68.

14 Gendelberg NR (2016) Diffusivity of Drug Actives in Transdermal Drug Delivery (TDD). MS Thesis Interdisciplinary Program in Materials Science \& Engineering, New Jersey Institute of Technology.

15 Benita S (1998) Submicron Emulsions in Drug Targeting and Delivery. Amsterdam: Harwood Academic.

16 http://www.permegear.com/franzatfaqs.htm

17 Wilson EJ (2011) "Three Generations of Transdermal Drug Delivery Systems. Three Generations of Transdermal Drug Delivery Systems. 